Shulyatnikova T. V., Tumanskiy V. O. Glutamine synthetase expression in the brain during experimental acute liver failure (immunohistochemical study). Journal of Education, Health and Sport. 2021;11(10): 342-356. eISSN 2391-8306. DOI http://dx.doi.org/10.12775/JEHS.2021.11.10.033

https://apcz.umk.pl/JEHS/article/view/JEHS.2021.11.10.033

https://zenodo.org/record/5807895

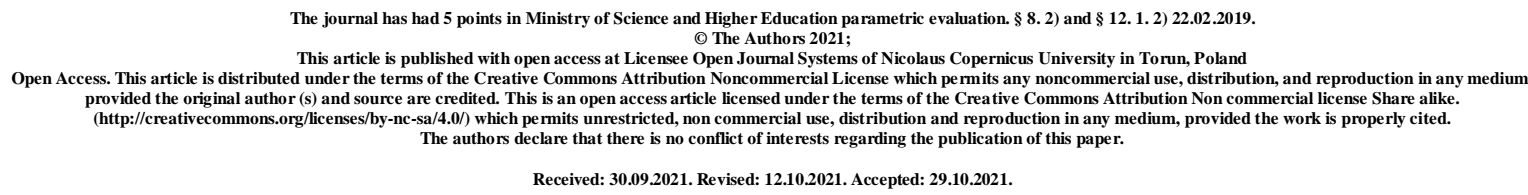

UDC 616.831:577.15]:616.36-008.64]-092.9-091.8-074/078

\title{
GLUTAMINE SYNTHETASE EXPRESSION IN THE BRAIN DURING EXPERIMENTAL ACUTE LIVER FAILURE (IMMUNOHISTOCHEMICAL STUDY)
}

\author{
T. V. Shulyatnikova, V. O. Tumanskiy
}

\section{Zaporizhzhia State Medical University, Ukraine}

Department of Pathological Anatomy and Forensic Medicine

Shulyatnikova T.V., MD, PhD, Associate Professor of the Department of Pathological Anatomy and Forensic Medicine, Zaporizhzhia State Medical University, Ukraine. shulyatnikova.tv@gmail.com +380958930149; ORCID ID: 0000-0002-0196-9935

Tumanskiy V.O., MD, DSc, Professor of the Department of Pathological Anatomy and Forensic Medicine, Vice-Rector for Research, Zaporizhzhia State Medical University, Honorary Scientist and Engineering Worker of Ukraine; ORCID ID: 0000-0001-8267-2350

\section{Abstract}

The aim of the study was to determine the immunohistochemical level of glutamine synthetase (GS) expression in different brain regions in the conditions of experimental acute liver failure in rats.

Materials and methods. The study was conducted in Wistar rats: 5 sham (control) animals and 10 rats with acetaminophen induced liver failure model (AILF). The immunohistochemical study of GS expression in the sensorimotor cortex, white matter, hippocampus, thalamus, caudate nucleus/putamen was carried out in the period of 12-24 $\mathrm{h}$ after acetaminophen treatment. 
Results. Beginning from the $6^{\text {th }}$ hour after acetaminophen treatment all AILF-animals showed the progressive increase in clinical signs of acute brain disfunction finished in 6 rats by comatose state up to $24 \mathrm{~h}$ - they constituted subgroup AILF-B, "non-survived". 4 animals survived until the $24 \mathrm{~h}$ - subgroup AILF-A, "survived". In the AILF-B group, starting from 16 to 24 hours after treatment, a significant (relative to control) regionally-specific dynamic increase in the level of GS expression was observed in the brain: in the cortex - by $307.33 \%$, in the thalamus - by $249.47 \%$, in the hippocampus - by $245.53 \%$, in the subcortical white matter - by $126.08 \%$, from $12^{\text {th }}$ hour - in the caudate nucleus/putamen, by $191.66 \%$; with the most substantive elevation of GS expression in the cortex: by 4.07 times.

Conclusion. Starting from the $16^{\text {th }}$ hours after the acetaminophen treatment (from the $12^{\text {th }} \mathrm{h}$ in the caudate nucleus/putamen region) and up to $24 \mathrm{~h}$, it is observed reliable compared to control dynamic increase in GS protein expression in the cortex, white matter, hippocampus, thalamus, caudate nucleus/putamen of the rat brain with the most significant elevation in the cortex among other regions. The heterogeneity in the degree of GS expression rising in different brain regions potentially may indicate regions more permeable for ammonia and/or other systemic toxic factors as well as heterogeneous sensitivity of brain regions to deleterious agents in conditions of AILF. Subsequently, revealed diversity in the GS expression reflects the specificity of reactive response of local astroglia in the condition of AILF-encephalopathy during specific time-period. The dynamic increase in the GS expression associated with impairment of animal state, indicates involvement of increased GS levels in the mechanisms of experimental acute hepatic encephalopathy.

\section{Key words: acute hepatic encephalopathy; astroglial reactivity; GS.}

\section{Introduction}

Advanced liver diseases are characterized by rising one of the most challenging neurocognitive disorder termed hepatic encephalopathy (HE), which occurs in approximately $30-45 \%$ of patients with cirrhosis [1]. The American Association for the Study of Liver Disease (AASLD)/European Association for the Study of the Liver (EASL) practice guidelines for HE defined hepatic encephalopathy as a «brain dysfunction caused by liver failure and/or portal-systemic shunting, which it manifests as a wide spectrum of neurological or psychiatric abnormalities ranging from subclinical alterations to coma» $[2,3]$. HE is potentially reversible and classified from Grade 0 - "minimal" to Grade IV - hepatic coma [4], determining poor patient prognosis and increased mortality [5]. Acute hepatic encephalopathy 
(AHE) is linked to acute liver failure (ALF) due to massive liver necrosis of different origin or fulminant viral hepatitis [6].

The pathophysiology of AHE still remains a largely unresolved issue and needs further clarification. It is widely considered that HE development is linked to the ammonia neurotoxicity [7, 8]. Impaired detoxification of ammonia due to severe liver dysfunction leads to increase circulating ammonia, crossing it the blood-brain barrier (BBB) and conditioning hyperammonemia within the brain parenchyma $[9,10]$. It is supposed that increased ammonia changes intracellular $\mathrm{pH}$, alter calcium homeostasis, neurotransmission and lead to excitotoxicity [6]. Among target cell population in the brain parenchyma for ammonia toxic effect astroglia appear to be on the central place as they considered the only ones containing glutamine synthetase and metabolize ammonia [11]. Pathogenic mechanisms of toxic action on astroglia include nitrative stress, downregulation of the gap-junction channel connexin-43, mitochondrial insufficiency, alteration of NO/cGMP pathway activity and y(+)LAT2mediated exchange of extracellular glutamine for intracellular arginine, senescence, and a wide list of other processes altered on genomic and/or molecular levels [6]. High levels of ammonia in the brain tissue lead to increased astroglial glutamine synthesis through activation of glutamine synthetase (GS), followed by astrocytic glutamine overload and their osmotic cytotoxic edema. Hence, astrocytes, representing the most numerous cellular fraction of brain volume, contribute substantially to the development of generalized cerebral edema-swelling, increased intracranial pressure resulting in hernial dislocation of the brain hemispheres and lethal outcome [7]. Mentioned mechanism formed the basis of the osmotic gliopathy theory which currently dominate in the explanation of the hyperammonemia induced cerebral edema [8]. CNS is enriched by glutamine and its synthesis is largely catalyzed by astroglial GS. Glutamine plays a key role in the neurotransmission being the precursor of the glutamate and gamma-aminobutyric acid (GABA). Briefly, as soon as glutamate is released in synaptic vesicles from presynaptic terminals, perisynaptic astrocytic processes capture excess glutamate via glutamate transporters and convert it into glutamine. Releasing glutamine from astrocytes into the extracellular space is followed by taking it back to neurons where it can be converted back to glutamate or GABA $[12,13]$. It was reported earlier that GS is upregulated in astroglia during acute hyperammonemia [6], while prolonged periods of increased systemic ammonia associated with controversial results concerning alteration of GS expression in the brain [14]. It is supposed that region- and context-dependent heterogeneity of astroglial population in healthy and diseased brain accompanied by diverse response of astrocytes to pathological stimuli [15]. As a consequence, it seems necessary to clarify supposed diverse 
response of different regional astroglial populations during acute hepatic encephalopathy in the experimental mode.

The aim of the study was to determine the immunohistochemical level of GS expression in different brain regions in the conditions of experimental acute liver failure in rats.

Materials and methods. The experiment was performed on Wistar rats, 200-300 g body weight. All procedures were conducted according to the European convention for the protection of vertebrate animals (Strasbourg, 18 March 1986; ETS No. 123) and the Directive 2010/63/EU. For induction of AHE type "A" ("Acute liver failure" - according to the American Association for the Study of Liver Disease updated guidelines), we used acetaminophen (paracetamol, N-acetyl-p-aminophenol [APAP]) induced liver failure (AILF) model $[16,17]$. For detailed characteristics of all steps of the experimental model see our previous paper [18]. Acetaminophen is one of the most common antipyretic drugs worldwide and it's overdosing determines the most frequent of ALF which often manifests in a hyperacute form [19]. Seeing the fact of acetaminophen intoxication causes ALF in rodents, AILF-model can be used for clarification the mechanisms of AHE similar to human one [16, 17].

Rats were divided into control group $(\mathrm{n}=5)$ and AILF-group $(\mathrm{n}=10)$. In the AILFgroup, after intraperitoneal acetaminophen injection, animals were examined for signs of changed major physiological parameters. Six rats were euthanized up to $24 \mathrm{~h}$ after the treatment by an intraperitoneal administration of sodium thiopental euthanasia solution due to the severe clinical symptoms of ALF up to coma and constituted the group "AILF-B" decompensated AILF (non-survived group). Rats that showed compensated clinical signs ( $\mathrm{n}=$ 4) and survived up to $24 \mathrm{~h}$ after the injection were designated to group "AILF-A" compensated AILF. In control (“AILF-C”) group, all animals survived up to 24 hours. All survived and control animals were euthanized by i.p. injection of sodium thiopental euthanasia solution at $24 \mathrm{~h}$. The brain and liver samples were processed according to standard procedures with formation of paraffin blocks. For general histopathological analysis hematoxylin-eosin stained sections were used. Immunohistochemical (IHC) study involved detection of immunopositive labels using rabbit polyclonal anti-GS primary antibody (Thermo Scientific, USA) and Ultra Vision Quanto Detection imaging system with diaminobenzidine (Thermo Scientific Inc., USA). The results of IHC reaction were assessed at magnification $\mathrm{x} 200$ in a standardized field of view (SFV) of the microscope Scope. A1 “Carl Zeiss" (Germany) using Jenoptik Progres Gryphax 60N-C1"1,0x426114 (Germany) 
camera and the program Videotest-Morphology 5.2.0.158 (Video Test LLC, RF). The expression of GS was assessed as a percentage of the relative area ( $\mathrm{S}$ rel., \%) of immunopositive labels to the total area of the tissue section in the SFV. For the comparative analysis of the GS expression were selected sensorimotor cortex, subcortical white matter, hippocampus, thalamus and caudate nucleus/putamen regions. Five SFV of each mentioned region were analyzed for each animal. Digital data were statistically processed by Statistica ${ }^{\circledR}$ for Windows 13.0 (StatSoft Inc., license № JPZ804I382130ARCN10-J) with evaluating median (Me), lower and upper quartiles (Q1; Q3). For comparison between groups MannWhitney and Kruskal-Wallis tests were used. The results were considered significant at $95 \%$ $(\mathrm{p}<0.05)$.

\section{Results}

At six hours after acetaminophen treatment all experimental animals showed the dynamic increase in clinical signs of acute brain disfunction finished by comatose state up to $24 \mathrm{~h}$ in 6 animals. Pathohistological study of the liver samples of all AILF-rats have evidenced wide foci of centrilobular necrosis, local hemorrhages and spread balloon dystrophy of hepatocytes, all characterized by dynamic aggravation over time of the experiment.

In the brain of control animals, at $24 \mathrm{~h}$ it was revealed heterogeneous level of GS expression among different regions with the highest level in the sensorimotor cortex -2.59 $(2.26 ; 3.63) \%$ and the lowest in the subcortical white matter $-0.23(0.17 ; 0.30) \%$ (Table 1$)$. GS-positive labeling in control brains was predominantly related to vascular astroglial endfeet processes and much lesser appeared to be found in parenchymal astrocytic processes (fig. 1). The latter trend was observed in all studied brain regions.

Table 1. The indicators of GS expression in different brain regions in animals of different experimental groups expressed in the percent of immune-positive labels in the SFV. Data are presented as median (Me) with lower and upper quartiles (Q1; Q3)

\begin{tabular}{|c|c|c|c|}
\hline Brain region & AILF-A & AILF-B & AILF-C \\
\hline Cortex & $7.04(6.15 ; 7.50) * \dagger$ & $10.55(7.71 ; 11.04) * \dagger$ & $2.59(2.26 ; 3.63)$ \\
\hline Subcortical white matter & $0.50(0.37 ; 1.34) *$ & $0.52(0.40 ; 1.39) *$ & $0.23(0.17 ; 0.30)$ \\
\hline Hippocampus & $3.22(2.91 ; 4.28) *$ & $3.87(3.04 ; 4.88) *$ & $1.12(0.53 ; 1.90)$ \\
\hline Thalamus & $3.10(2.23 ; 3.24) *$ & $3.32(2.11 ; 3.90) *$ & $0.95(0.23 ; 1.65)$ \\
\hline Caudate/putamen & $2.33(2.17 ; 3,72) *$ & $2.44(2.17 ; 4.16) *$ & $0.84(0.34 ; 1.23)$ \\
\hline
\end{tabular}


Reliable differences in indicators compared to the control animals $(p<0.05)$ are marked with an asterisk (*). Reliable differences between CLP-A and CLP-B groups in the same brain region $(p<0.05)$ are marked with the dagger $(\dagger)$; "CLP-A" - survived; "CLP-B" non-survived; "CLP-C" - control.

\begin{tabular}{|l|l|l|}
\hline & \\
Fig. 1. GS expression in the cortex of the & Fig. 2. GS expression in the cortex of the \\
control rat (AILF-C group) 24 h after the & non-survived rat (AILF-B group) 24 h after \\
sham procedure. (anti-GS, Thermo & the AILF procedure. (anti-GS, Thermo \\
Scientific, USA). 200. & Scientific, USA). 200.
\end{tabular}

Histopathological studying of ICH-stained sections of all studied brain regions of rats after AILF-procedure revealed substantive increase in GS-positive labeling, which was predominantly associated to astroglial bodies cytoplasm and was distributed more evenly over all types of astroglial processes (fig. 2).

In the experimental AILF-A and AILF-B animals the alteration of GS expression was heterogenous among different brain regions with the highest increase in the cortex. Wherein both survived and non-survived rats showed substantive reliable elevation in GS expression in all studied regions compart to control indicators of the same region. Moreover, the indicators of GS expression in all notes regions was higher in AILF-B animals compared to AILF-A, while with no statistical validity of differences $(\mathrm{p}>0.05)$, except cortical region (Table 1$)$.

Thus, in the non-survived AILF-B group the relative area of $\mathrm{GS}^{+}$expression displayed the most prominent increase in the cortical region comparing to control, respectively: 10.55 $(7.71 ; 11.04) \%$ and $2.59(2.26 ; 3.63) \%, p<0.05$, that was equal to $307.33 \%$ or 4.07 -fold increase if compare medians values of indicators. At $24 \mathrm{~h}$ of the experiment, AILF-A group either showed reliable increase cortical GS expression - $7.04(6.15 ; 7.50) \%$, although, it was reliably lower compared to AILF-B group (p <0.05) (Table 1).

After cortical values, thalamic and hippocampal regions of AILF-B group was the next most notable locations in the brain where $\mathrm{GS}^{+}$expression increase substantially: 3.32 (2.11; 
$3.90) \%$ (3.49-fold or $249.47 \%$ compared to control) and $3.87(3.04 ; 4.88) \%$ (3.45-fold or $245.53 \%$ compared to control) respectively ( $\mathrm{p}<0.05)$. In AILF-A group, thalamic and hippocampal increased expression was also significant and reliable compared to control, although did not differ reliably comparing to AILF-B values ( $p>0.05)$ (Table 1).

Less substantial elevation of GS expression in AILF-B rats was belonged to caudate/putamen region, although values were reliably higher than in control indicators: 2.44 $(2.17 ; 4.16) \%$ vs. $0.84(0.34 ; 1.23)$ respectively, with an excess of $191.66 \%$ or 2.91 times compared to control.

The least pronounced but reliable increase of GS expression in non-survived animals was found in subcortical white matter $-0.52(0.40 ; 1.39) \%$ - that exceeded control values on $126.08 \%$ or by 2.26 times.

In AILF-B animals, during the post-injection period the increasement of GS expression in the brain performed growing dynamics (figs. 3-7). Depending on the time after acetaminophen injection when animals displayed symptoms of decompensated of ALF and were sacrificed, values of GS also differed between regions, while the highest values of the GS was found $24 \mathrm{~h}$ after the injection in all the studied regions (figs. 3-7). Starting from $16 \mathrm{~h}$ of the experiment, all sacrificed animals of AILF-B group displayed reliable increase in GS values compared to control group, except the caudate/putamen region where increasement of indices reached statistical validity already by 12 hours after injection (figs. 3-7).

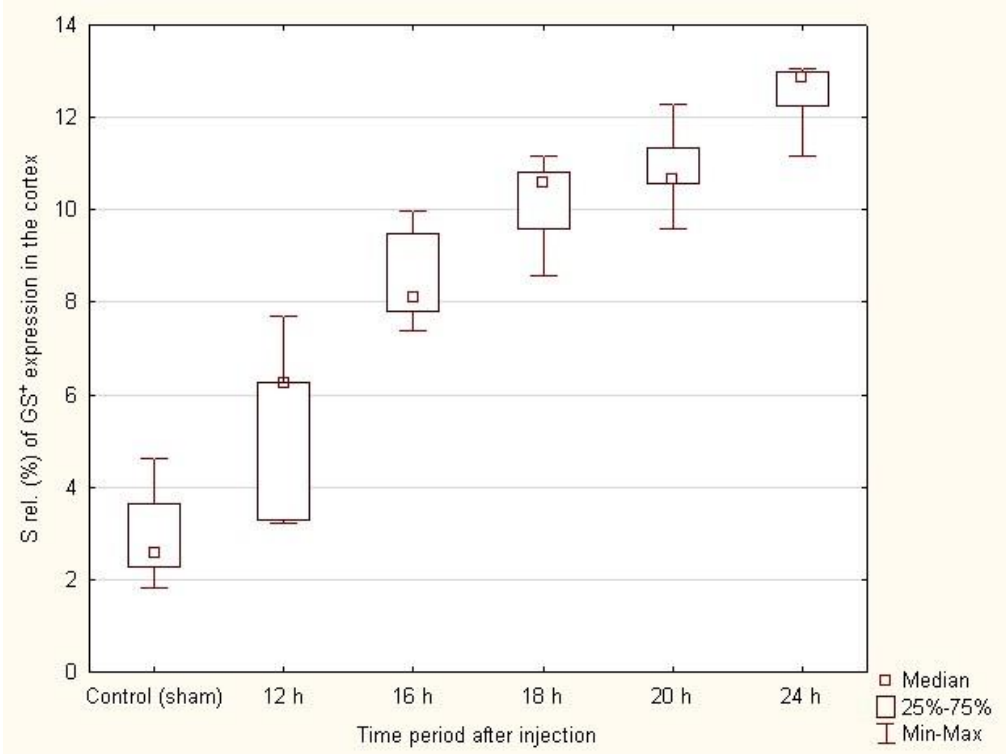

Fig. 3. Dynamics of the relative area of $\mathrm{GS}^{+}$expression (in the microscope SFV, \%) in the cerebral cortex of AILF-B rats after acetaminophen treatment 


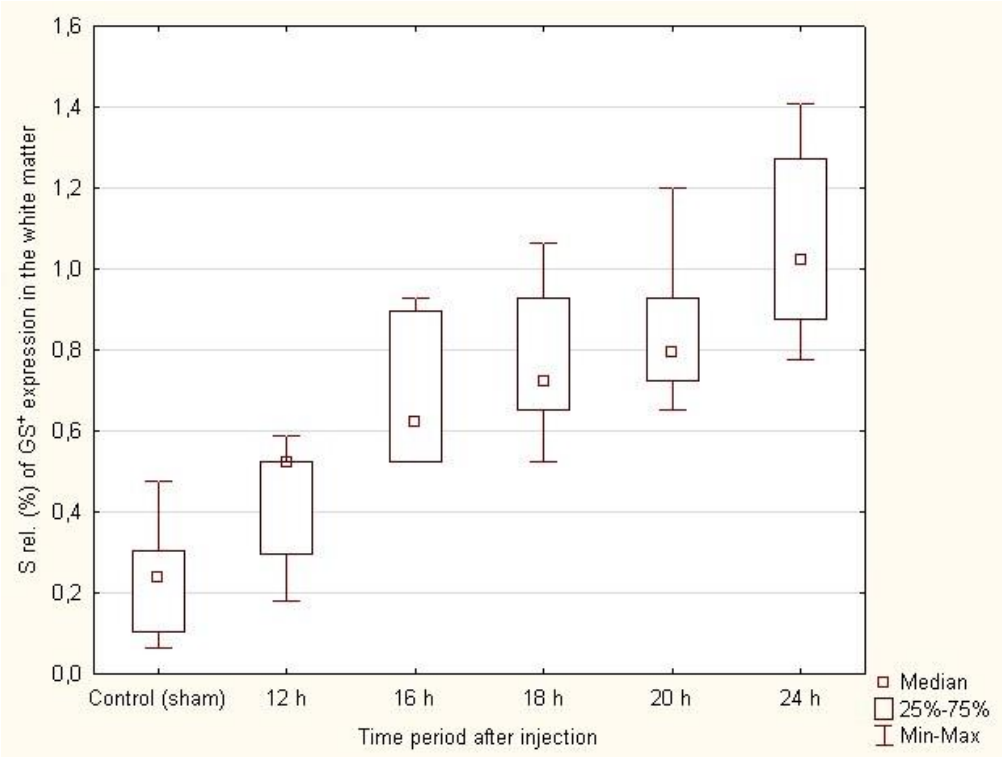

Fig. 4. Dynamics of the relative area of $\mathrm{GS}^{+}$expression (in the microscope $\mathrm{SFV}, \%$ ) in the subcortical white matter of AILF-B rats after acetaminophen treatment

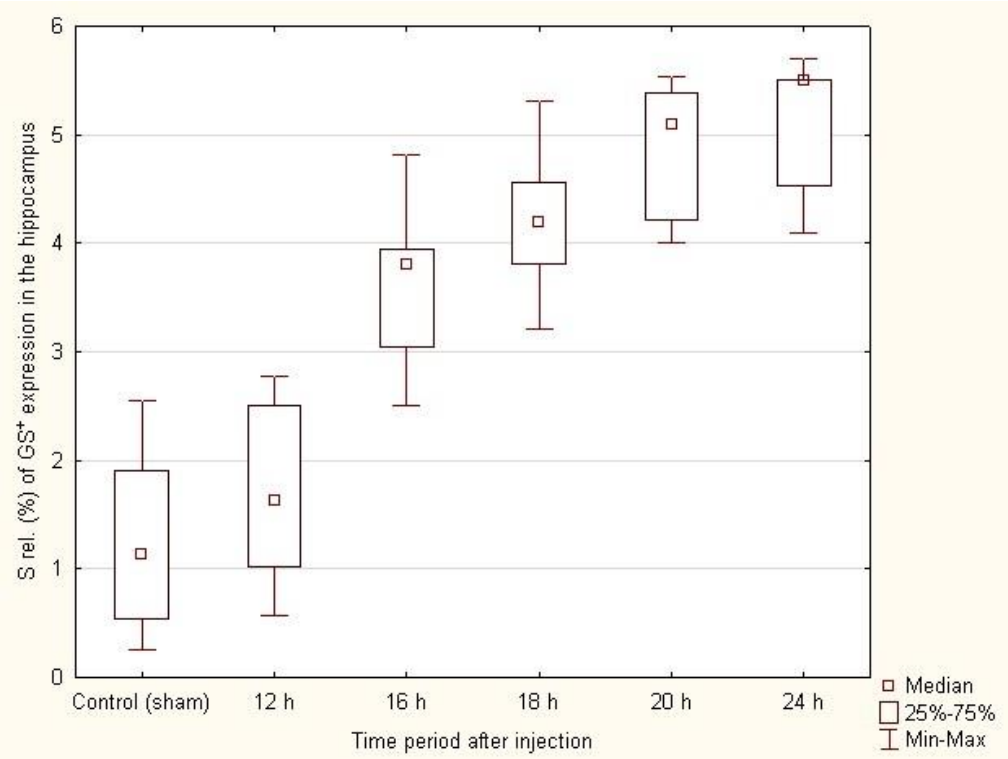

Fig. 5. Dynamics of the relative area of $\mathrm{GS}^{+}$expression (in the microscope $\mathrm{SFV}, \%$ ) in the hippocampus of AILF-B rats after acetaminophen treatment

In sum, after acetaminophen treatment, the highest values of GS expression were typical for the AILF-B animals in all studied brain regions with the most substantial rising in the cortex, thalamus and hippocampus compared to control. Significant exponential elevation of the GS expression was revealed at $16 \mathrm{~h}$ after injection in all studied regions, except caudate/putamen, where it gained reliable increase as early as $12 \mathrm{~h}$ after AILF-procedure. 


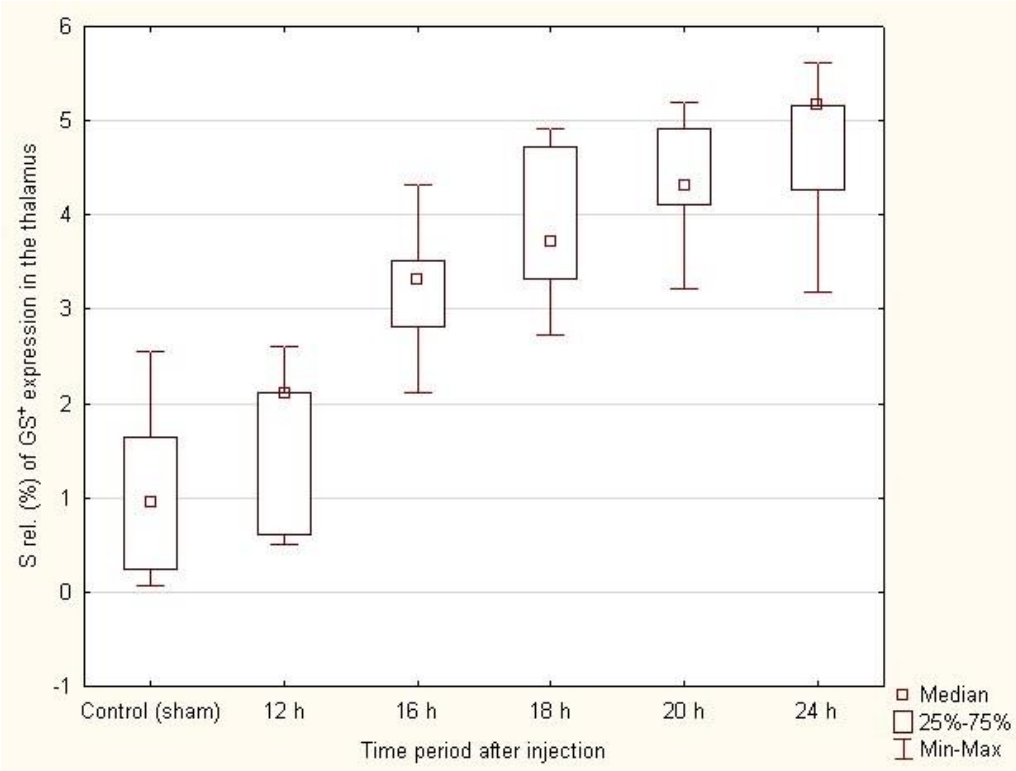

Fig. 6. Dynamics of the relative area of $\mathrm{GS}^{+}$expression (in the microscope SFV, \%) in the thalamus of AILF-B rats after acetaminophen treatment

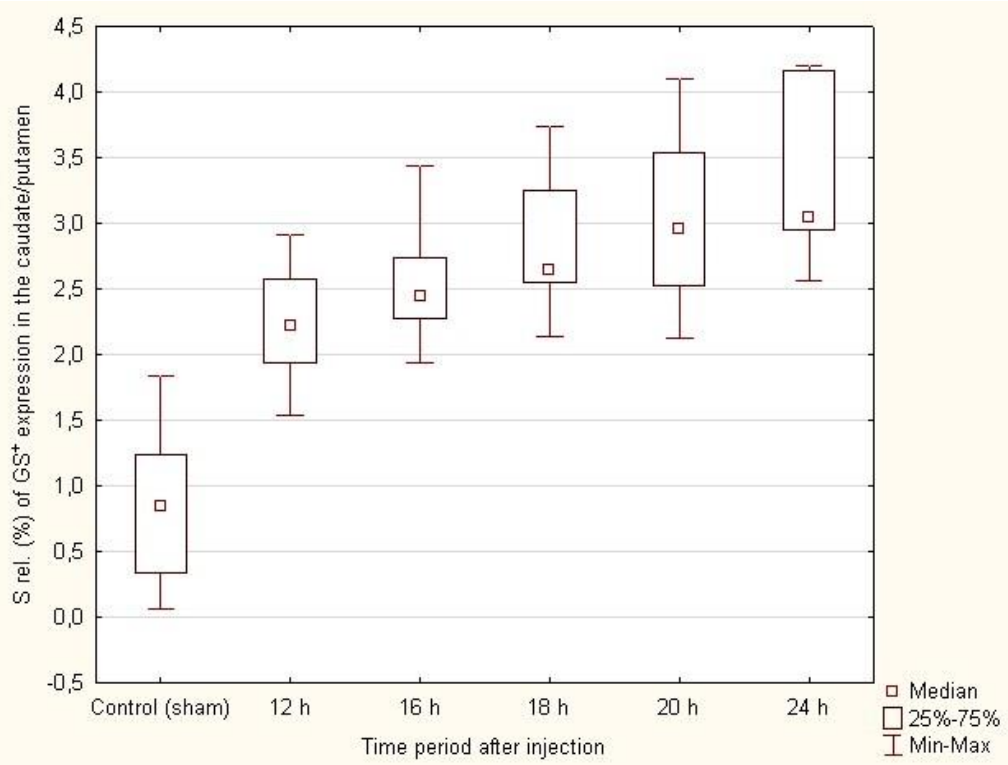

Fig. 7. Dynamics of the relative area of $\mathrm{GS}^{+}$expression (in the microscope SFV, \%) in the caudate nucleus/putamen of AILF-B rats after acetaminophen treatment

\section{Discussion}

Cerebral edema is considered to be a key criterion of AHE, but it is not the only sign, given the influence of other mechanisms. The majority of previous studies on pathogenesis of cerebral edema in ALF are based on experiments using astrocytes cultures treated by ammonia, animal models of acute liver damage, or translational studies of patients with ALF [11]. Considering the evidence provided by numerous studies, ammonia plays a crucial role in 
the mechanisms of AHE in the condition of ALF, and astroglia appears to be a principle site of cellular dysfunction in the brain in these conditions [20]. Early studies of AHE have been resulted in the classic «glutamine/osmolyte hypothesis» postulated that systemic and brain hyperammonemia leads to accumulation of glutamine in astrocytes (despite ammoniamediated oxidative stress and protein tyrosine nitration of astroglial GS with its inhibition/inactivation), and followed by astrocyte swelling, cytotoxic brain edema without significant neuronal death $[21,13,22]$. Nevertheless, further studies have challenged glutamine substrate as the main osmotic reason of astrocyte swelling and brain edema in AHE making classical viewpoints controversial and debatable. Thus, it was confirmed by many studies using ammonia exposure to astrocyte culture and experimental HE, that brain glutamine level does not correlate clearly with the degree of astroglial swelling and brain edema [11]. Among other the most common alternative mechanisms suggesting the link between glutamine and brain edema in hyperammonemia was the concept named "Trojan Horse hypothesis" [7, 11]. According to this mechanism, accumulated inside astrocytes glutamine further transported to the mitochondria and undergoes hydrolysis by glutaminase with subsequent ammonia production. Thereby glutamine acts as a «Trojan Horse» carrying ammonia into the astrocyte mitochondria and initiates mitochondrial energetic dysfunction with opening the mitochondrial permeability transition pore [7]. The latter cause mitochondrial membrane depolarization, energy failure, malfunction or insufficiency of energy-dependent ion transporters, subsequent oxidative stress and cytotoxic cellular edema of astrocytes [14].

GS is physiologically expressed in extrahepatic tissues such as skeletal muscle, kidneys, brain, adipose tissue and several others, which are collectively responsible for nearly $1 / 3^{\text {rd }}$ of the total capacity of the organism to ammonia detoxification via the GS [23]. It was found experimentally that brain GS is essential for surviving and the significance of astroglial GS was evidenced by knockout technics in very recent studies. Selective deletion of GS in the whole mouse brain results in a 14-fold decrease in cortical glutamine accompanied by 1.6fold increase in cortical ammonia in mice [24]. Zhou Y. and colleagues were succeeded to selectively delete astroglial GS in the neocortex and hippocampus using mice of the Emx1_IRES Cre line. In this study, adult experimental animals exhibited altered locomotive activity, neurodegeneration and spontaneous seizures [25]. In contrast, deletion of GS in other organs did not cause serious phenotype abnormalities in animals [13].

Spodenkiewicz M. et al. in their review reported about three patients with rare recessive inborn partial loss of GS function in all GS expressed organs which clinically 
presented brain atrophy, severe neonatal epileptic encephalopathy and early death from multiple organ failure [26].

Both human and animal studies have suggested a role of GS in the mechanisms of epileptogenesis, where decreased glutamate-glutamine-GABA cycling between neurons and astroglia may contribute to excessive excitability. Furthermore, in human studies, the decrease in GS expression and/or activity was shown for brain areas with signs of reduced neuronal density - in epileptogenic hippocampus in patients with mesial temporal lobe epilepsy, amygdala in some patients with neocortical epilepsy, in the tumor tissue of patients with secondary epilepsy [27]. Experimental inhibition or deletion of GS in the entorhinalhippocampal brain region causes mesial temporal lobe epilepsy-like syndrome characterized by spontaneous hippocampal-onset seizures and loss of hippocampal neurons in animals [27].

In our study we immunohistochemically evidenced that in the condition of AILFmodel there is early $(16 \mathrm{~h})$ reliable increase in the expression level of GS protein in the brain cortex, white matter, hippocampus, thalamus and at $12 \mathrm{~h}$ - in the caudate nucleus/putamen regions. The most prominent elevation of the GS expression level in the cortex might suppose that among other brain regions, cortex is characterized by more substantial ammonia load and exposure, as well as more pronounced action of other deleterious factors altering the function of glutamine-glutamate shuttle. Considering strong heterogeneity of astroglial populations through different regions in healthy and diseased brain, the diverse expression levels of GS enzyme in AILF might reflect special reactive features of different astroglial populations among studied brain regions. The earliest reliable increase of GS expression in caudate/putamen region might propose the most rapid ammonia entry into the brain parenchyma in this region as well as the faster reactivity of local astroglia on the action of hyperammonemia. Continuing this thought, the lowest elevation indexes of GS expression in the white matter characterizes the local astroglial population and/or this region as the most unreactive and/or protected from the ammonia exposure. The higher values of GS expression in the cortex of non-survived animals compared to survived group, as well as the maximal levels of GS at $24 \mathrm{~h}$, when non-survived animals progressively showed extinction of vital functions and aggravation of coma state, might indicate the active involvement of GS in the mechanisms of decompensation of acute encephalopathy, which is typical for AILF.

Both deficiency and upregulation of GS with subsequent deficit or overproduction and accumulation of glutamine in the brain may lead to highly deleterious effects on astroglial and neuronal community causing both overexcitability and deceleration states depending on the level of GS activity, astroglial microenvironment, developmental ontogenetic period of the 
organism, specific brain region, period of the disease, etc. These specific signatures may suggest different therapeutic approaches in each case. Moreover, considering the known complexity of the course of any pathological state including HE, one should comprehensively take into account the wide range of the noted influencing factors while analyzing specific clinical cases or experimental data.

\section{Conclusion}

Starting from the 16th hours after the acetaminophen treatment (from the 12th $\mathrm{h}$ in the caudate nucleus/putamen region) and up to $24 \mathrm{~h}$, it is observed reliable compared to control dynamic increase in GS protein expression in the cortex, white matter, hippocampus, thalamus, caudate nucleus/putamen of the rat brain with the most significant elevation in the cortex among other regions. The heterogeneity in the degree of GS expression rising in different brain regions potentially may indicate regions more permeable for ammonia and/or other systemic toxic factors as well as heterogeneous sensitivity of brain regions to deleterious agents in conditions of AILF. Subsequently, revealed diversity in the GS expression reflects the specificity of reactive response of local astroglia in the condition of AILF-encephalopathy during specific time-period. The dynamic increase in the GS expression associated with impairment of animal state, indicates involvement of increased GS levels in the mechanisms of experimental AHE.

\section{References}

1. Hirode, G., Vittinghoff, E., \& Wong, R. J. (2019). Increasing Burden of Hepatic Encephalopathy Among Hospitalized Adults: An Analysis of the 2010-2014 National Inpatient Sample. Digestive diseases and sciences, 64(6), 1448-1457. https://doi.org/10.1007/s10620-019-05576-9

2. Vilstrup, H., Amodio, P., Bajaj, J., Cordoba, J., Ferenci, P., Mullen, K. D., Weissenborn, K., \& Wong, P. (2014). Hepatic encephalopathy in chronic liver disease: 2014 Practice Guideline by the American Association for the Study of Liver Diseases and the European Association for the Study of the Liver. Hepatology (Baltimore, Md.), 60(2), 715735. https://doi.org/10.1002/hep.27210

3. Amodio, P., \& Montagnese, S. (2021). Lights and Shadows in Hepatic Encephalopathy Diagnosis. Journal of clinical medicine, 10(2), 341. https://doi.org/10.3390/jcm10020341

4. Weissenborn K. (2019). Hepatic Encephalopathy: Definition, Clinical Grading and Diagnostic Principles. Drugs, 79(Suppl 1), 5-9. https://doi.org/10.1007/s40265-018$\underline{1018-\mathrm{Z}}$ 
5. Ferenci P. (2017). Hepatic encephalopathy. Gastroenterology report, 5(2), 138-147. https://doi.org/10.1093/gastro/gox013

6. Jayakumar, A. R., \& Norenberg, M. D. (2018). Hyperammonemia in Hepatic Encephalopathy. Journal of clinical and experimental hepatology, 8(3), 272-280. https://doi.org/10.1016/j.jceh.2018.06.007

7. Shulyatnikova T. \& Shavrin V. (2017). Modern view on hepatic encephalopathy: basic terms and concepts of pathogenesis. Pathologia, 14(3), 371-380 (41). https://doi.org/10.14739/2310-1237.2017.3.118773

8. Brusilow, S. W., Koehler, R. C., Traystman, R. J., \& Cooper, A. J. (2010). Astrocyte glutamine synthetase: importance in hyperammonemic syndromes and potential target for therapy. Neurotherapeutics: the journal of the American Society for Experimental NeuroTherapeutics, 7(4), 452-470. https://doi.org/10.1016/j.nurt.2010.05.015

9. Dasarathy, S., Mookerjee, R. P., Rackayova, V., Rangroo Thrane, V., Vairappan, B., Ott, P., \& Rose, C. F. (2017). Ammonia toxicity: from head to toe? Metabolic brain disease, 32(2), 529-538. https://doi.org/10.1007/s11011-016-9938-3

10. Ochoa-Sanchez, R., Tamnanloo, F., \& Rose, C. F. (2021). Hepatic Encephalopathy: From Metabolic to Neurodegenerative. Neurochemical research, 46(10), 2612-2625. https://doi.org/10.1007/s11064-021-03372-4

11. Liotta, E. M., \& Kimberly, W. T. (2020). Cerebral edema and liver disease: Classic perspectives and contemporary hypotheses on mechanism. Neuroscience letters, 721, 134818. https://doi.org/10.1016/j.neulet.2020.134818

12. Walls, A. B., Waagepetersen, H. S., Bak, L. K., Schousboe, A., \& Sonnewald, U. (2015). The glutamine-glutamate/GABA cycle: function, regional differences in glutamate and GABA production and effects of interference with GABA metabolism. Neurochemical research, 40(2), 402-409. https://doi.org/10.1007/s11064-014-1473-1

13. Zhou, Y., Eid, T., Hassel, B., \& Danbolt, N. C. (2020). Novel aspects of glutamine synthetase in ammonia homeostasis. Neurochemistry international, 140, 104809. https://doi.org/10.1016/j.neuint.2020.104809

14. Jaeger, V., DeMorrow, S., \& McMillin, M. (2019). The Direct Contribution of Astrocytes and Microglia to the Pathogenesis of Hepatic Encephalopathy. Journal of clinical and translational hepatology, 7(4), 352-361. https://doi.org/10.14218/JCTH.2019.00025

15. Batiuk, M. Y., Martirosyan, A., Wahis, J., de Vin, F., Marneffe, C., Kusserow, C., Koeppen, J., Viana, J. F., Oliveira, J. F., Voet, T., Ponting, C. P., Belgard, T. G., \& Holt, 
M. G. (2020). Identification of region-specific astrocyte subtypes at single cell resolution. Nature communications, 11(1), 1220. https://doi.org/10.1038/s41467-019-14198-8

16. McGill, M. R., Williams, C. D., Xie, Y., Ramachandran, A., \& Jaeschke, H. (2012). Acetaminophen-induced liver injury in rats and mice: comparison of protein adducts, mitochondrial dysfunction, and oxidative stress in the mechanism of toxicity. Toxicology and applied pharmacology, 264(3), 387-394. https://doi.org/10.1016/j.taap.2012.08.015

17. Mossanen, J. C., \& Tacke, F. (2015). Acetaminophen-induced acute liver injury in mice. Laboratory animals, 49(1 Suppl), 30-36. https://doi.org/10.1177/0023677215570992

18. Shulyatnikova T, Shavrin V. (2021). Mobilisation and redistribution of multivesicular bodies to the endfeet of reactive astrocytes in acute endogenous toxic $\begin{array}{llll}\text { encephalopathies. } & \text { Brain } & \text { Research, } & \text { 1751:147174. }\end{array}$ https://doi.org/10.1016/j.brainres.2020.147174.

19. Mitchell, R. A., Rathi, S., Dahiya, M., Zhu, J., Hussaini, T., \& Yoshida, E. M. (2020). Public awareness of acetaminophen and risks of drug induced liver injury: Results of a large outpatient clinic survey. PloS one, 15(3), e0229070. https://doi.org/10.1371/journal.pone.0229070

20. Butterworth R. F. (2015). Pathogenesis of hepatic encephalopathy and brain edema in acute liver failure. Journal of clinical and experimental hepatology, 5(Suppl 1), S96-S103. https://doi.org/10.1016/j.jceh.2014.02.004

21. Häussinger, D., Görg, B., Reinehr, R., \& Schliess, F. (2005). Protein tyrosine nitration in hyperammonemia and hepatic encephalopathy. Metabolic brain disease, 20(4), 285-294. https://doi.org/10.1007/s11011-005-7908-2

22. Aldridge, D. R., Tranah, E. J., \& Shawcross, D. L. (2015). Pathogenesis of hepatic encephalopathy: role of ammonia and systemic inflammation. Journal of clinical and experimental hepatology, 5(Suppl 1), S7-S20. https://doi.org/10.1016/j.jceh.2014.06.004

23. Hakvoort, T. B., He, Y., Kulik, W., Vermeulen, J. L., Duijst, S., Ruijter, J. M., Runge, J. H., Deutz, N. E., Koehler, S. E., \& Lamers, W. H. (2017). Pivotal role of glutamine synthetase in ammonia detoxification. Hepatology (Baltimore, Md.), 65(1), 281-293. https://doi.org/10.1002/hep.28852

24. He, Y., Hakvoort, T. B., Vermeulen, J. L., Labruyère, W. T., De Waart, D. R., Van Der Hel, W. S., Ruijter, J. M., Uylings, H. B., \& Lamers, W. H. (2010). Glutamine synthetase deficiency in murine astrocytes results in neonatal death. Glia, 58(6), 741-754. $\underline{\text { https://doi.org/10.1002/glia.20960 }}$ 
25. Zhou, Y., Dhaher, R., Parent, M., Hu, Q. X., Hassel, B., Yee, S. P., Hyder, F., Gruenbaum, S. E., Eid, T., \& Danbolt, N. C. (2019). Selective deletion of glutamine synthetase in the mouse cerebral cortex induces glial dysfunction and vascular impairment that precede epilepsy and neurodegeneration. Neurochemistry international, 123, 22-33. https://doi.org/10.1016/j.neuint.2018.07.009

26. Spodenkiewicz, M., Diez-Fernandez, C., Rüfenacht, V., Gemperle-Britschgi, C., \& Häberle, J. (2016). Minireview on Glutamine Synthetase Deficiency, an Ultra-Rare Inborn Error of Amino Acid Biosynthesis. Biology, 5(4), 40. https://doi.org/10.3390/biology5040040

27. Sandhu, M., Gruenbaum, B. F., Gruenbaum, S. E., Dhaher, R., Deshpande, K., Funaro, M. C., Lee, T. W., Zaveri, H. P., \& Eid, T. (2021). Astroglial Glutamine Synthetase and the Pathogenesis of Mesial Temporal Lobe Epilepsy. Frontiers in neurology, 12, 665334. https://doi.org/10.3389/fneur.2021.665334 